\title{
An Approach of Neural Network For Electrocardiogram Classification
}

\author{
Mayank Kumar Gautam*, Vinod Kumar Giri \\ Department of Electrical Engineering, Madan Mohan Malaviya University of Technology, \\ Gorakhpur, India \\ *Corresponding author, e-mail: prince240791@ gmail.com
}

\begin{abstract}
ECG is basically the graphical representation of the electrical activity of cardiac muscles during contraction and release stages. It helps in determination of the cardiac arrhythmias in a well manner. Due to this early detection of arrhythmias can be done properly. In other words we can say that the bio-potentials generated by the cardiac muscles results in an electrical signal called Electro-cardiogram (ECG). It acts as a vital physiological parameter, which is being used exclusively to know the state of the cardiac patients. Feature extraction of ECG plays a vital role in the manual as well as automatic analysis of ECG. In this paper the study of the concept of pattern recognition of ECG is done. It refers to the classification of data patterns and characterizing them into classes of predefined set. The analysis ECG signal falls under the application of pattern recognition. The ECG signal generated waveform gives almost all information about activity of the heart. The ECG signal feature extraction parameters such as spectral entropy, Poincare plot and Lyapunov exponent are used for study in this paper.This paper also includes artificial neural network as a classifier for identifying the abnormalities of heart disease.
\end{abstract}

Keywords: ECG, ANN, Feature extraction, Feature classification, Arrhythmia.

Copyright $(2) 2016$ APTIKOM - All rights reserved.

\section{Introduction}

Electrocardiography gives information of the electrical activity of the cardiac muscles. Biosignals which are usually non-stationary signals may occur randomly in the time-scale. Hence, for the effective diagnosis, the ECG signal pattern and heart rate variability should be observed over several hours. Because of the volume of the data being enormous due to long time recording, the analysis of it is tedious and also time consuming. Therefore, automatic computer-based examination and classification of cardiac diseases can be very helpful in diagnostic [1]. The frequency range of an ECG signal lies in between $0.05-100 \mathrm{~Hz}$ and its magnitude lies in the range of $1-10 \mathrm{mV}$. It is been characterized by five peaks and valleys labeled as $\mathrm{P}, \mathrm{Q}, \mathrm{R}, \mathrm{S}$ and $\mathrm{T}$ as shown in Figure 1 . The performance of any automatic ECG analyzer depends majorly on the accurate and reliable detection of the QRS segmentation part, as well as $\mathrm{T}$ and $\mathrm{P}$ waves. The detection of the QRS segmentation part is the crucial task in automatic ECG signal analysis. Because, once the QRS segmentation part has been acknowledged a more comprehensive assessment of ECG signal can be performed that includes the heart rate, the ST segment etc. The normal beats have the P-R interval usually in the range of 0.12-0.2 seconds whereas the QRS interval lies in the range of 0.04-0.12 seconds. The division of ECG is basically in two phases as depolarization of the cardiac muscles and repolarisation of the cardiac muscles. The depolarization phases include the $\mathrm{P}$ wave i.e, atrial depolarization and QRS-wave i.e, ventricles depolarization. The repolarisation phases include the T-wave and U-wave i.e, ventricular repolarisation [2-6]. Malfunction in the signaling in the myocardium results in the heart to pump blood less effectively and deteriorates proper conduction process of the heart [4]. Hence, the early detection of arrhythmias is very helpful for living a durable and reliable life as well as improves early detection of arrhythmias. Generally, the standard ECG signals are categorized into three different groups and shown in Figure 1.

a. Waves-deviations from the isoelectric line i.e, the baseline voltage. They are named successively: P, Q, R, S, T, U.

b. Segments-isoelectric lines time duration between waves.

c. Intervals- time duration which include segments and waves. 


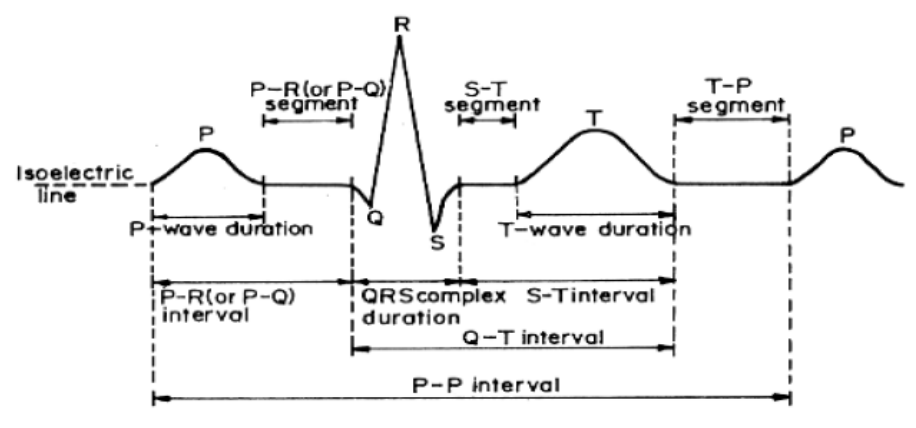

Figure 1. ECG signal

An effective as well as efficient analysis of ECG signal depends upon accurate and reliable detection of the P, Q, R, S and T waves. The detection of QRS complex is the most important task in automated ECG signal analysis because once the QRS complex is being identified, a more meticulous assessment of ECG signal is doable by including the heart rate and ST segment [5-6]. Number of algorithms have been developed and also discussed in the current paper for early detection as well as classification of these ECG signals.

\section{Research Work \& Literature Review At A Glance}

Enormous algorithms have been developed for detection, feature extraction as well as classification of ECG signals. Ramli et al. [7] have used a cross correlation analysis technique for extracting the vital features from 12 lead ECG signal. Using the cross correlation techniques the values being identified can be used to predict the type of arrhythmias. Tadejko and Rakowski [8], introduced an automatic classifier with K-self-organizing maps (SOM) and LVQ algorithms. This paper compares the QRS complexes for classification and then preprocesses the ECG morphology features. The performance of algorithms is to examine the beats are either in the form of normal or arrhythmia condition.

Table 1. Various abnormalities and their characteristic feature

\begin{tabular}{ccc}
\hline SR.NO. & NAME OF THE ARRHYTHMIA & CHARACTERSITIC FEATURES \\
\hline 1 & Dextrocardia & Inverted P-wave \\
2 & Tachycardia & R-R interval $<0.6 \mathrm{sec}$ \\
3 & Bradycardia & R-R interval $>1 \mathrm{sec}$ \\
4 & Hyperkalemia & Tall T-wave and absence of P-wave \\
5 & Myocardial & Ischemia Inverted T-wave \\
6 & Hypercalcaemia & QRS interval $<0.1$ sec \\
7 & Sinoatrial block & Complete drop out of a cardiac cycle \\
8 & Sudden cardiac death & Irregular ECG \\
\hline
\end{tabular}

$\mathrm{Xu}$ et al. in [9] proposed the SVM algorithm for ECG QRS complex detection as well as the RR interval determination. They have used the Slope vectors for the extraction of features of ECG wave and also the non-linear amplification have been used to improve $\mathrm{S} / \mathrm{N}$. This paper introduces high accuracy and fast response to find the QRS detection.

Manpreet Kaur and Arora [10] anticipated the K-means clustering along with Squared Euclidean distance for the ECG signals analysis. For ECG feature extraction the parameters identified as wave shape, duration and amplitude. Using $\mathrm{K}$-means clustering technique, clustered $\mathrm{K}$ is summed and minimizes to centroid distance. With the help of this technique, first phase gives information about the points that are resigned to the closet cluster around the centroid. The second phase gives information where values are self-reconciled. The success rate of classification for different datasets of MIT-BIH are analyzed.

F. de Chazal et.al [23] determined the PVC from the normal beats. For feature extraction of ECG signals, the combination of the morphological based features and time interval based features have been proposed. For the classification of ECG signal, the MLP with different number of hidden layers and algorithm according to radial basis function neural network (RBFNN) and probabilistic neural network

APTIKOM J. CSIT Vol. 1, No. 3, 2016 : 119-127 
(PNN) is used. The simulation results show that about $97.14 \%$ for classification is achieved. For simulation purpose, the MIT-BIH arrhythmia database is used.

S. Mitra et.al [11] determined a rough-set theory for analysis of ECG signal. A rule-based roughset decision system is developed from time-domain features to make an inference engine for arrhythmia detection. This technique helps to optimize rules for cardiac- arrhythmia detection, by which the complexity of Neural Networks (NN) can be omited. Currently, the system is tested with three types of ECG datasets as Normal, Myocardial Ischemia, and Myocardial Infarction and the accuracy of all these types is obtained for both the trained and untrained dataset.

Castro et al. [12] anticipated a wavelet transform algorithm for feature extraction of an ECG signal and identification of abnormal heartbeats. The ECG signal is first denoised by a soft or hard threshold and then each cycle is simplified into a coefficient vectors using the optimal wavelet function (OWF). The analyzed ECG signal coefficients are divided into the $\mathrm{P}, \mathrm{Q}, \mathrm{R}, \mathrm{S}$ and $\mathrm{T}$ to obtain a feature vector of the signal cycles.

Nazmy et al [13] determined the ANFIS algorithm for classification of ECG wave. The ECG feature extraction is done using the Independent Component Analysis (ICA). The power spectrum and input is being provided by the RR interval of ECG signal. In this, the ECG signals been classified are Normal Sinus Rhythm (NSR), Premature Ventricular Contraction (PVC), Atrial-Premature Contraction (APC), Ventricular Tachycardia (VT), Ventricular Fibrillation (VF) and Supraventricular Tachycardia (SVT) with the help of ANFIS approach, the classification accuracy is also obtained.

Alan and Nikola in [14] had introduced Chaos theory for feature extraction of ECG signals. Various methods comes under the Chaos theory, including correlation dimension, phase space and attractors, central tendency measure, spatial filling index, and approximate entropy are also determined. Yuksel and Bekir [17] have represented ANN to classify the ECG arrhythmias.

Zhu et.al, [18] determined the application of ANN for ECG abnormality detection. In this paper, to analyze the performance, the SOM network, BP and LVQ network were used and overall accuracy of these networks has been acheived. [19,20,21] also presented a comparison of how the neural networks classify the patterns from training data and recognizes if testing data holds that patterns of ECG signal.

El-Khafif et al. [19] anticipted ANN model to make a diagnosis of the ischemic heart disease from normal ECG signals. Feed forward Multilayer perceptron neural network is used with error back propagation learning algorithm.

Hosseini et al. [22] have anticipated the use of a two-stage feed forward neural network (FF-NN) for ECG signal classification in which they have elected two network architectures on the basis of one stage and two stages feed forward neural networks (FF-NN) to recognize heart arrhythmias.

Manimegalai et al. [28] have determined the use a discrete wavelet transform based system for detection and feature extraction of $\mathrm{P}, \mathrm{Q}, \mathrm{R}, \mathrm{S}$ and $\mathrm{T}$ waves and in which they found that this technique consumes less computational time and has better accuracy for ECG classification, analysis and characterization of normal and abnormalities of ECG. In [25,26,27] the neuro-fuzzy technique has been proposed to configure the experimental data.

Golpayegani and Jafari [29] proposed anticipated a comparative evaluation of recital of ANFF with MLP neural networks and it has been found that the time of training required for the ANFF was much shorter than time required by MLP.

Owis et al. [30] have determined the correlation dimension and largest Lyapunov exponent parameters to sculpt the chaotic nature of different classes of ECG signals. The anticipated implementations were used to compute these features belongs to five different types of ECG signal taken from the MIT-BIH Arrhythmia Database. The results are premeditated to detect statistically major differences among different arrhythmia types. Finally, statistical classification techniques are applied to assess the possibility of detecting and to classify arrhythmia using such parameters.

\section{Methodology Involved}

The introduction of neural network was firstly in 1943 by the famous neurophysiologist Warren McCulloch and logician Walter Pits. ANNs are biologically inspired networks which has many applications in the field such as in pattern recognition, classification etc. The decision making procedure of the ANN is great and logical, based on the features of input patterns, and is greatly applied in the area of classification of biomedical data. Typically, multilayer feed forward neural networks (MPNN) can be trained as non-linear classifiers using the generalized back propagation algorithm (BPA) [31-33]. The back propagation algorithm is a type of supervised learning algorithm, in which a mean square error 
(MSE) function is defined, and the basic aim of the learning process is to reduce the overall system error to a minimum.

The technique of classification is done by logical and accurate neural network algorithm called Back Propagation Network (BPN). The learning algorithm of the multilayer perceptions requires a differentiable activation function but frequently used is logical function (non-linear, monotonic, increasing, differentiable). The term back propagation states that the backward propagation of an error signal through the network. After propagating a pattern through the feed forward network, the output pattern is compared with a given target and the error of each output unit is calculated. The error of each output unit is propagated backwards to the input layer - back propagation. Finally the errors of the units are used to modify the weights

The back-propagation neural network (BPNN) usually allows practical attainment of each input/output mapping information within each multilayer networks. Basically, BPNN executes the algorithm of gradient descent search to minimize the mean square error (MSE) between the preferred output and the actual output of the network by adjusting their weights. Back propagation (BPNN) algorithm is highly in usage because in most classification problems the reason is that the characteristics of the generalized data rule $[33,36]$.

It is a forward multi-layer network, which trains the network by the use the error backpropagation algorithm. The back propagation network algorithm (BPN) was proposed by Rumelhart et al in 1986, because of its simple structure, multiadjustable parameters, much training algorithm and good operational performance by these are all this network got a wide range of practical application. The network structure of the three-layer BP neural network is shown in Figure 2.

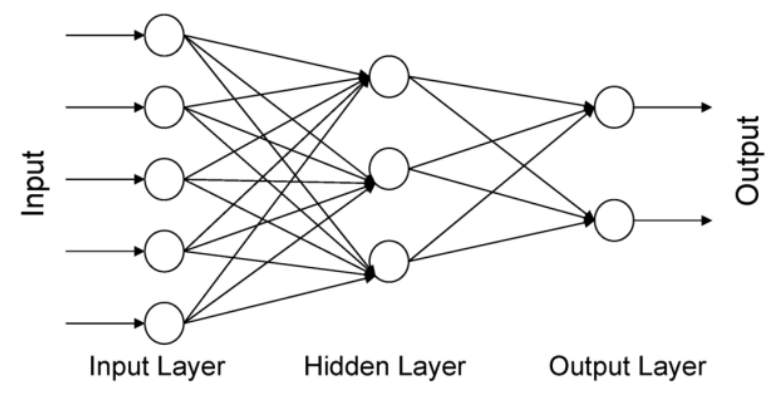

Figure 2. Structure of ANN

Which show that this neural network consists of an input layer and middle layer (hidden layer) and an output layer. In this, there is a complete connectivity between the upper and lower layers and there are no connections between neurons in each layer. That the input layer of the signal needs to spread towards to hidden layer nodes and transformed by the function, then transmission of the input signal of hidden layer nodes to the output layer nodes. Typically that the transfer function of BP neural network is Sigmoid Type differentiable function, which can attain random non-linear mapping between the input and output, from this result this type of network has been wide applications in the pattern recognition, function approximation and other areas. $[11,36]$

\section{Parameters of Aritifcial Neural Network \\ 4.1. Special Entropy}

Spectral entropy specifies and simplifies the spectral complexity of the time series. There is a variety of spectral transformations exist. Of these varieties, the Fourier transformation (FT) is the most widely and greatly used technique and which gives the power spectral density (PSD) can be obtained. Application of Shannon's channel entropy produces an estimate of the spectral entropy, where entropy is given by

$$
\mathrm{H}=\sum_{\mathrm{f}} \mathrm{P}_{\mathrm{f} \log }\left(\frac{1}{\mathrm{P}_{\mathrm{f}}}\right)
$$

where $\mathrm{P}_{\mathrm{f}}$ denotes the PDF value at frequency $\mathrm{f}$.

APTIKOM J. CSIT Vol. 1, No. 3, 2016 : 119-127 
The spectral entropy $H(0<=H<=1)$ describes the complexity of the HRV signal. The $H$ was computed for the various types of cardiac signal.

\subsection{Poincare Plot Geometry}

Poincare plot geometry is a technique which explains the nature of R-R interval fluctuations. It is basically a graph of each R-R interval which is plotted against the next interval [30]. Poincare plot analysis is an emerging quantitative-visual method in which the plot shape is categorized into functional classes that indicate the degree of heart failure in a subject. The Poincare plot can be analyzed quantitatively by the calculations of the standard deviations of the distances of the R-R (i) to the lines $y=$ $\mathrm{x}$ and $\mathrm{y}=-\mathrm{x} \div 2 * \mathrm{R}-\mathrm{R}_{\mathrm{m}}$, where $\mathrm{R}-\mathrm{R}_{\mathrm{m}}$ is the mean of all $\mathrm{R}-\mathrm{R}(\mathrm{i})$. Standard deviations are denoted as SD1 and SD2. SD1 is related to the fast beat-to-beat variability in the data, whereas SD2 described the longterm variability of R-R (i). In order to describe the relationship between these components, the ratio of SD1/SD2 can also be computed. Figure 3 shows the Poincar6 plot of a normal subject.

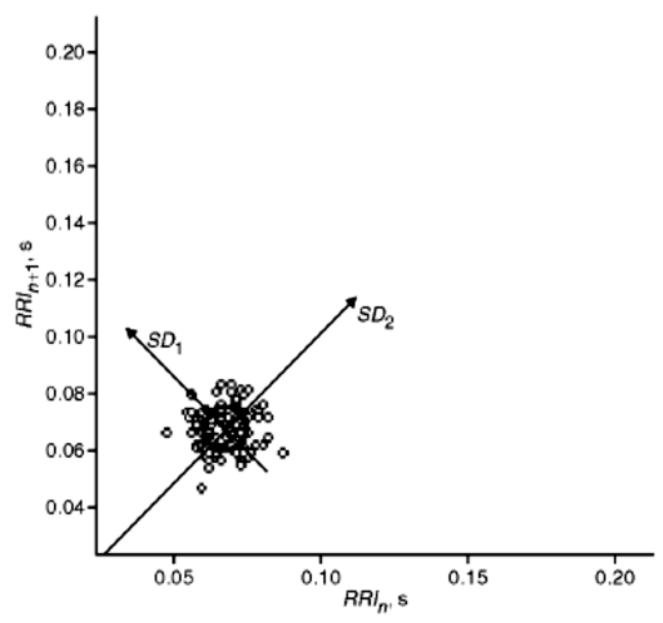

Figure 3. Poincare Plot Geometry

\subsection{Lyapunov Exponent}

The Lyapunov exponent $\lambda$ is a measure of the rate at which the trajectories separate one from another. A negative exponent implies that the orbits approach a common fixed point [5]. A zero exponent means the orbits maintain their relative positions; they are on a stable attractor. Finally, a positive exponent implies that the orbits are on a chaotic attractor. For two points in a space $X_{0}$ and $X_{0}+\Delta X_{0}$, that are function of time and each of which will generate an orbit in that space using some equations or system of equations, then the separation between the two orbits $\Delta \mathrm{x}$ will also be a function of time. This partition is likewise an element of the area of the beginning value and has the structure $\Delta \mathrm{x}\left(\mathrm{X}_{0}, \mathrm{t}\right)$. For a riotous information set, the capacity $\Delta \mathrm{x}\left(\mathrm{X}_{0}, \mathrm{t}\right)$ will act capricious.

\section{Experimental Layout}

\subsection{Feature Extraction}

Wavelet transform is a periodic scale representation that has been utilized effectively as a part of a wide scope of utilizations, specifically signal compression. As of late, Wavelets have been connected to a few issues in Electro cardiology, including data compression, examination of ventricular late possibilities, and the location of ECG characteristic points. The Wavelet transform is a straight operation that breaks down the signal into various scales identified with recurrence parts and examinations every scale with a specific determination. The WT utilizes a brief span interim for assessing higher frequencies and quite a while interim for lower frequencies. Because of this property, high frequency components of short term can be watched effectively by Wavelet Transform. One of the benefits of the Wavelet Transform is that it can break down signs at different resolutions, which permits precise component extraction from non-stationary signs like ECG. A group of breaking down wavelets in the time recurrence 
area is acquired by applying a scaling component and an interpretation element to the fundamental mother wavelet.

Wavelet change of a sign $\mathrm{f}(\mathrm{t})$ is characterized as the entirety of over unsurpassed of the sign duplicated by scaled, moved forms of the wavelet capacity $\psi$ and is given $b$

$$
\begin{aligned}
& \mathrm{w}(\mathrm{a}, \mathrm{b})=\int_{+\infty}^{-\infty} \mathrm{f}(\mathrm{t}), \varphi \mathrm{a}, \mathrm{b}(\mathrm{t}) \mathrm{dt} \\
& \varphi a,(t)=\frac{1}{\sqrt{\mathrm{a} \varphi^{\mathrm{n}}}}\left(\frac{\mathrm{t}-\mathrm{b}}{\mathrm{a}}\right)
\end{aligned}
$$

The DWT is adequate for most of the applications and for the recreation of the sign. The DWT gives enough data, and it gives a huge decrease in the calculation time. Here, we have discrete capacity $\mathrm{f}$ (n) and the meaning of DWT is given by:

$$
\mathrm{w}(\mathrm{a}, \mathrm{b})=\mathrm{c}(\mathrm{j}, \mathrm{k})=\sum_{n=z} f(n) \mathrm{w}_{\mathrm{j}, \mathrm{k}(\mathrm{n})}
$$

where $\varphi_{\mathrm{j}, \mathrm{k}(\mathrm{n})}$ is a discrete wavelet transform

$$
\varphi_{\mathrm{j}, \mathrm{k}(\mathrm{n})=2^{2}}^{\stackrel{\mathrm{j}}{\mathbf{c}}} \varphi\left(\mathrm{z}^{-\mathrm{j}} \mathrm{n}-\mathrm{k}\right)
$$

In the DWT examinations, the signal at various frequency bands and at various resolutions is deteriorated into a 'coarse estimation' and 'point by point data'. Two arrangements of capacities are utilized by the DWT, the scaling capacities. Figure 4 shows levels of ECG Decompostion.

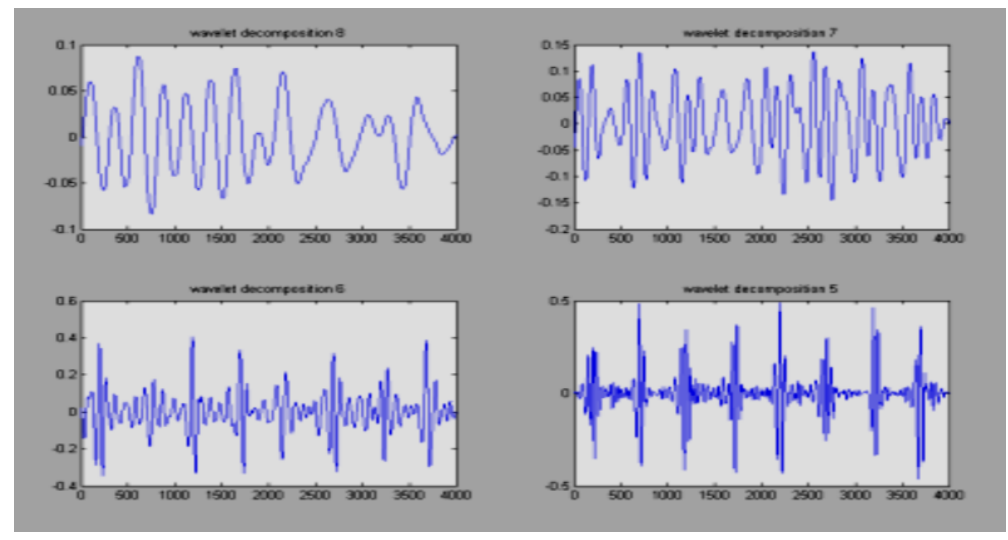

Figure 4. Levels of ECG Decompostion

(Connected with the low pass channel) and the wavelet capacities (connected with the high pass channel). The sign is sifted by going it through progressive high pass and low pass channels to acquire variants of the sign in various recurrence groups. The essential thought behind wavelets is to dissect as per scale. These are capacities that fulfill certain numerical necessities and are utilized as a part of speaking to information or different strategies. I calculations process information at various scales or resolutions. In the event that the sign with a substantial window, we would see gross elements. Additionally signal with a small window, then notice that have a small element. Figure 5 shows levels of ECG decomposition. 


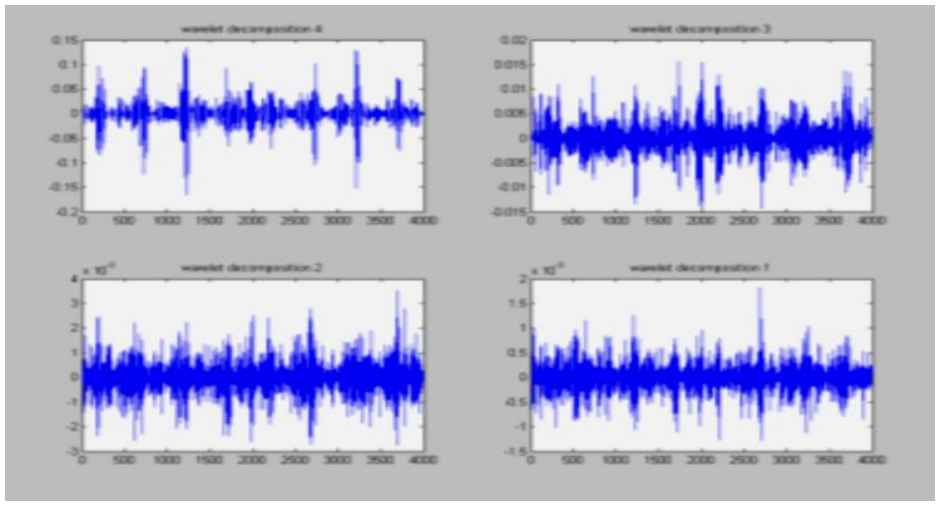

Figure 5. Levels of ECG Decomposition

\subsection{Analysis using BPNN Algorithm}

At that point the order is finished by Back Propagation Network. The learning calculation multilayer discernments requires a differentiable actuation capacity, much of the time is logistic capacity utilized (non-direct, monotonic, expanding, differentiable). The term back proliferation implies the regressive spread of a blunder signal through the system. Subsequent to proliferating an example through the system - encourage forward, the yield example is contrasted and a given target and the mistake of every yield unit is ascertained. This blunder is proliferated in reverse to the data layer - back spread. At long last the blunders of the units are utilized to adjust the weights.

\section{Results and Discussion}

The data are collected from MIT-BIH database. In this paper 34 signals are used for training and from this 15 signals are taken for testing.

Our techniques accomplishes the separation in the normal and abnormal heart rate, these are analysis by back propagation neural system. Our technique demonstrates ongoing application execution parameter can precisely judge that the exactness.

Table 2. Performance Parameters

\begin{tabular}{ccc}
\hline METHOD APPLIED & SPECIFICITY & ACCURACY \\
\hline BACK PROPAGATION NEURAL NETWORK(BPNN) & $100 \%$ & $93.3 \%$ \\
\hline
\end{tabular}

\section{Future Scope and Enhacements}

As the ECG analysis plays a vital role in determination and discrimination of many types of cardiac arrhythmias [37-38]. In addition, the further enhancement observes on utilizing different method that provides higher accuracy in feature extraction and classification. Hence, the neural networks proved a milestone in the analysis of the ECG and provide great achievement in the diagnosis of cardiac diseases.

\section{Conlusion}

ECG signals required for investigation are gathered from Physionet MIT-BIH arrhythmia database the database contains 48 records, each containing two-channel ECG signals for 30 min length of time chose from 24-hr recordings of 47 distinct people. The techniques were produced under MATLAB. Subsequent to the utilization of wavelet change in electro cardiology is generally new field of examination, numerous methodological perspectives (Choice of the mother wavelet, estimations of the scale parameters) of the wavelet strategy will require further examinations with a specific end goal to enhance the clinical helpfulness of this novel sign handling framework. Simultaneously analytic and prognostic noteworthiness of wavelet strategies in different fields of electro cardiology should be set up in vast clinical studies. Extremely straightforward and quick dependable techniques are proposed in this paper. This system is anything but difficult to perform and it needn't bother with any complex scientific estimations, for example, Fourier strategies, cross-connection. The ANN classifier was nourished by three 
parameters to be spectral entropy, Poincare plot geometry and biggest Lyapunov type (LLE) got from the heart rate signs are talked about.

\section{Acknowledgement}

This work is supported by University Grant Commission (UGC), New Delhi, India under Major Research Project (MRP) scheme. This work has been carried out in Electrical Engineering Department of MMM University of Technology, Gorakhpur, India.

\section{References}

[1] C.T. Lin, C.F. Juang, (2001) "An adaptive neural fuzzy filter and its applications," IEEE Transactions On Systems, MAN, And Cybernetics, VOL. 27, NO. 4, 1103-1110.

[2] Jenisha.J.Hannah and Suja Priyadharsini,2012. Patient Adaptive ECG Beat Classifier using Repetition Detection Approach Enhanced by Neural Networks, International Conference on Computing and Research and Control Engineering (ICCCE 2012), 12 \& 13 ISBN:978-1-4675- 2248-9.

[3] K .O. Gupta and Dr. P. N. Chatur, 2012. ECG Signal Analysis and Classification using Data Mining and Artificial Neural Networks, International Journal of Emerging Technology Advanced Engineering, ISSN:22502459, 2(1) 56-60.

[4] J. T. Catalano, Guide to ECG analysis, Lippincott, $2^{\text {nd }}$ edition

[5] Bucolo M., Grazia F. D., Sapuppo F., Nikolic D., Vuksanovic B., 2009. Multidimensional Analysis toward the Identification of ECG Nonlinear Dynamics. PHYSCON 2009. Catania, Italy.

[6] Y.C. Yeha, and W. J. Wang, 2008. QRS complexes detection for ECG signals The Difference Operation Method (DOM), Computer methods and programs in biomedicine, vol. 9, pp. 245-254.

[7] A. B. Ramli, and P. A. Ahmad, "Correlation analysis for abnormal ECG signal features extraction", $4^{\text {th }}$ National Conference on Telecommunication Technology, 2003. NCTT 2003 Proceedings, pp. 232- 237

[8] P. Tadejko, and W. Rakowski, "Mathematical Morphology Based ECG Feature Extraction for the Purpose of Heartbeat Classification, 6th International Conference on Computer Information Systems and Industrial Management Applications",2007, CISIM '07, pp. 322-327.

[9] Xiaomin Xu, and Ying Liu, 2004 "ECG QRS Complex Detection Using Slope Vector Waveform (SVW) Algorithm", Proceedings of the 26th Annual International Conference of the IEEE EMBS, pp. 3597- 3600.

[10] Manpreet Kaur and A.S. Arora" Unsupervised Analysis of Arrhythmias using K-means Clustering" (IJCSIT) International Journal of Computer Science and Information Technologies, Vol. 1 (5) , 2010, 417- 419.

[11] S. Mitra, and B. B. Chaudhuri, M. Mitra," A rough set based inference engine for ECG classification",2006,IEEE Trans. Instrum. Meas., 55(6): 2198-2206.

[12] B. Castro, D. Kogan, and A. B. Geva, 2000. "ECG feature extraction using optimal mother wavelet".The 21st IEEE Convention of the Electrical and Electronic Engineers in Israel, pp. 346-350.

[13] T. M. Nazmy, H. El-Messiry and B. Albokhity," Adaptive Neuro-Fuzzy Inference System for Classification of Ecg Signals, Journal of Theoretical and Applied Information Technology",2009.

[14] Alan Jovic, and Nikola Bogunovic, 2007 "Feature Extraction for ECG Time-Series Mining based on Chaos Theory ", Proceedings of 29th International Conference on Information Technology Interfaces.

[15] Raushan Ara Dilruba, Nipa Chowdhury, Farhana Ferdousi Liza, Karmakar,K.C., "Data pattern recognition Using neural network with back propagation training”, pp 451-455, 2006.

[16] Philip de Chazal, Maria O'Dwyer and Richard B.Reilly, "Automatic Classification of Heartbeats Using ECG Morphology and Heartbeat Interval Features", pp 1196 - 1206, 2004.

[17] Yuksel Ozbay and Bekir Karlik, "A Recognition of ECG Arrhythmias Using Artificial Neural Networks", pp. $1680-1683,2001$.

[18] K.Zhu, P. D. Noakes and A.D.P. Green, "ECG Monitoring with Artificial Neural Networks",pp.205 - 209, 1991.

[19] El-Khafif S. H. and El-Brawany M. A., "Artificial Neural Network-Based Automated ECG Signal Classifier", ISRN Biomedical Engineering, 2013.

[20] Mr.Deshmukh Rohan, Dr. A. J. Patil, "Layered Approach for ECG betas Classification utilizing Neural Network functions",2012,International Journal of Engineering Research and Applications (IJREA) ISSN:22489622.,2(6): 1495-1500

[21] S. Osowaki, T.H. Linh, "ECG beat recognition using fuzzy hybrid neural network", 2001, IEEE Trans. Biomed. Eng. 48 (11) 1265-1271.

[22] Hosseini H.G., Luob D. and Reynolds K. J, 2006 "The comparison of different feed forward neural network architectures for ECG signal diagnosis",Medical Engineering \& Physics.28: 372-378.

[23] F. de Chazal and R. B. Reilly," A patient adapting heart beat classifier using ECG morphology and heartbeat interval features",2006, IEEE Trans. Biomed. Eng., 53(12): 2535-2543.

[24] S.Y. Foo, G. Harvey, A. Meyer-Baese, "Neural network based ECG pattern recognition",2002, Eng. Appl. Artificial Intelligence, 15, 353-360.

APTIKOM J. CSIT Vol. 1, No. 3, 2016 : 119-127 
[25] V. Pilla, H.S. Lopes, "Evolutionary training of a neuro-fuzzy network for detection of a $P$ wave of the $E C G$ ",1999,Proceeding of the third international conference on computational intelligence and multimedia applications, New Dehli, India, 102-106.

[26] M. Engin, S. Demirag, (2003), "Fuzzy-hybrid neural network based ECG beat recognition using three different types of feature sets," Cardiovasc. Eng. Int. J. 3 (2) 71-80.

[27] Ranganathan G., Rangarajan R. and Bindhu V., 2011. "Evaluation of ECG Signals for Mental Stress Assessment using Fuzzy Technique". International Journal of Soft Computing and Engineering (IJSCE). 1(4): 195-201

[28] Manimegalai P., Bharathi P. and Thanushkodi K., 2012. Real Time Implementation of Analysis of ECG Characteristic Points Using Discrete Wavelets. Global Journal of researches in engineering Electrical and electronics engineering. 12(1).

[29] Glayol Nazari Golpayegani, Amir Homayoun Jafari. A novel approach in ECG beat recognition using adaptive neural fuzzy filter. J. Biomedical Science and Engineering, 2009, 2, 80-85.

[30] Owis M. I., Abou-Zied A. H., Youssef A. M. and Kadah Y. M.,"Study of Features Based on Nonlinear Dynamical Modeling in ECG Arrhythmia Detection and Classification”. IEEE Transactions on Biomedical Engineering. 49 (7).

[31] M. Bahoura, M. Hassani, and M. Hubin, "DSP implementation of wavelet transform for real time ECG wave forms detection and heart rate analysis", Comput. Methods Programs Biomed, vol.52, no.1, (1997), pp.35-44.

[32] Y. H. Hu, W. J. Tompkins, J. L. Urrusti and V. X. Afonso, "Applications of artificial neural networks for ECG signal detection and classification”, J .Electrocardiology, vol.26, (1993), pp. 66-73.

[33] http://www.physionet.org/physiobank/database/mitdb/.

[34] R. Ganguli, "Noise and Outlier Removal from Jet Engine Health Signals using Weighted FIR Median Hybrid Filters", Mechanical Systems and Signal Processing, vol.16, no.6, (2002), pp.967-978.

[35] C. Li, C. Zheng, Proc. Annual Int. Conf. IEE Eng. in Med. \& Biol. Soc., (1993); San Diego, California.

[36] Neural Network Toolbox. Available: http://www.mathworks.com.

[37] L. Khadra, A. Fraiwan and W. Shahab, "Neural-wavelet analysis of cardiac arrhythmias", Proceedings of the WSEAS International Conference on Neural Network and Applications (NNA '02), (2002); Interlaken, Switzerland.

[38] S. Pachekhiya and A. K. Wadhwani, "Disease Diagnosis Of Heart Muscles Using Error Back propagation Neural Network", International Journal of Engineering Science and Technology. 